Published in final edited form as:

Pediatr Pulmonol. 2016 June ; 51(6): 650-657. doi:10.1002/ppul.23441.

\title{
Highlights from the 2015 North American Cystic Fibrosis Conference
}

\author{
Edith T. Zemanick, MD ${ }^{1,{ }^{*}}$, Thida Ong, MD², Cori L. Daines, ${ }^{2}{ }^{3}$, Elisabeth P. Dellon, MD ${ }^{4}$, \\ Marianne S. Muhlebach, $\mathbf{M D}^{4}$, and Charles R. Esther Jr., MD, $\mathbf{P h D}^{4}$ \\ ${ }^{1}$ Department of Pediatrics, University of Colorado School of Medicine, Aurora, Colorado \\ 2Division of Pulmonary and Sleep Medicine, Department of Pediatrics, Seattle Children's \\ Hospital, University of Washington, Seattle, Washington \\ ${ }^{3}$ Department of Pediatrics, University of Arizona College of Medicine, Tucson, Arizona \\ ${ }^{4}$ Division of Pulmonology, Department of Pediatrics, University of North Carolina at Chapel Hill, \\ Chapel Hill, North Carolina
}

\section{Summary}

The 29th Annual North American Cystic Fibrosis Conference was held in Phoenix, Arizona on October 8-10, 2015. Abstracts were published in a supplement to Pediatric Pulmonology. ${ }^{1}$ In this review, we summarize presentations in several of the topic areas addressed at the conference. Our goal is to provide an overview of presentations with relevance to emerging or changing concepts in several areas rather than a comprehensive review. Citations from the conference are by first author and abstract number or symposium number, as designated in the supplement.

\section{Keywords}

cystic fibrosis; clinical trials; antibiotic therapy; quality improvement; CFTR modulators; personalized medicine

\section{PATHOPHYSIOLOGY OF CYSTIC FIBROSIS (CF) LUNG DISEASE}

The theme of personalized or precision medicine was prevalent throughout the basic science sessions. This is exemplified by the emphasis on "theratyping," grouping cystic fibrosis transmembrane conductance regulator (CFTR) gene mutations by their mechanistic response to different therapeutics. ${ }^{2}$ The response of missense and in-frame deletion mutants to CFTR correctors and potentiators was discussed (Harness-Brumley et al., S17.1), with further data provided in workshop sessions (Cai et al., 3; Sorscher et al., 199). A common theme was that responses to CFTR modulators are mutation specific, with differential responses among various CFTR alleles not always related to functional class. For example, the VX-809 corrector designed to address CFTR misprocessing was more effective with the R117H mutation (a conductance mutation) than N1303K (a misprocessing mutation), whereas the

\footnotetext{
"Correspondence to: Edith T. Zemanick, MD, 13123 E. 16th Ave. B-395, Aurora, CO 80045. edith.zemanick@ childrenscolorado.org. Conflicts of interest: None.
} 
gating mutations S1251N and G551D had differential responses to the potentiator VX-770 (Cholon et al., 6). Thus, the historic division of CFTR mutations into different functional classes is not necessarily predictive of responses to specific CFTR modulator therapies, with implications for development and use of these drugs.

Novel approaches toward improving CFTR protein function were also explored. Many CFTR mutations, including F508del, experience excessive protein degradation via the ubiquitin-dependent degradation pathway. The ubiquitin-activating enzyme inhibitor PYR-41 was shown to enhance the corrector activity of VX-809 (Goeckeler-Fried et al., 10), and new inhibitors of ubiquitin E3 ligases appear to rescue F508del CFTR (Chung et al., 13). Different approaches for other types of mutations were also discussed, such as readthrough agents for premature termination codons. These drugs permit generation of full length protein, but tend to insert certain amino acids (tryptophan, cysteine, or arginine) effectively creating missense mutations. Activity of CFTR proteins generated by readthrough therapies depends upon the location of the premature stop codon (Pranke et al., 12 and 153), and activity of some corrected proteins can be increased by CFTR potentiators (Mutyam et al., 219). These findings suggest that combination of a readthrough agent plus a CFTR potentiator may be needed for clinical efficacy. ${ }^{3}$ These basic science studies form the underpinning for future, novel clinical therapeutic approaches in CF.

All of these studies demonstrate the need for a precision medicine approach to determine the optimal drugs and drug combinations for specific CFTR mutations. Several groups are examining the potential of organoids to test individual patients' responses to various drugs. Organoids are hollow spheres of epithelial cells formed from cells obtained from intestinal or airway tissue biopsies. CFTR activity can be assessed by whether the spheres grow or shrink in response to drugs that alter CFTR function (Amaral, S9.2). Several groups highlighted the potential utility of this approach (Ramalho et al., 18; Berkers et al., 210; Kwok et al., 61; Sorio et al., 163; Boecking et al., 188; Caldrer et al., 259). While the use of organoids represents an exciting approach to precision medicine in $\mathrm{CF}$, all of the investigators noted the need for further work to ensure that the ex vivo responses in organoids are predictive of clinical benefit.

Testing of new therapies requires robust animal models, but these efforts are hindered by the fact that the CF knockout mouse model does not develop significant pulmonary disease. The development of the F508del CF rat model (Lambert et al., 85) represents a significant advance, in part, because the use of CRISPR technology to engineer a specific CFTR mutation potentially paves the way for mutation specific animal models. Importantly, the CF rat appears to exhibit a pulmonary phenotype with mucus accumulation and difficulty clearing intratracheally administered Pseudomonas (Birket et al., 139), potentially providing a much needed small animal model of CF lung disease.

Insights were also derived from other animal models. A relationship between lowered airway surface liquid (ASL) $\mathrm{pH}$ and increased mucus viscosity with greater impact in smaller airways was shown in the CF pig (Tang et al., 91; Li et al., 92). Pharmacological correction of reduced ASL pH improved bacterial killing and ASL viscosity, potentially identifying a new therapeutic strategy (Shah et al., 97). The glandular mucus transport 
defects in the airways of the "European" CF pig, which are consistent with findings in intestinal mucosa of the CF mouse, were reviewed in a symposium session (Hansson et al., $\mathrm{S} 2.2$ ). These findings highlight the potential benefit of improving mucus properties with hydrating agents, reducing agents, or improved CFTR function (Button, S2.1; Rowe et al., $\mathrm{S} 2.4$ ). The CF ferret led to new insights in non-pulmonary phenotypes including bone development (Havasi et al., 593), glucose intolerance (Yi et al., 595), and macrophage function (Keiser et al., 88). The multitude of available CF animal models including mouse, rat, ferret, and pig represents a boon for CF researchers, particularly since some of these models appear to develop lung disease. However, variations in phenotype highlight the limitations of animal models and the need to verify the relevance of pathophysiological mechanisms in humans.

Challenges remain in understanding the role of the microbiome and interbacterial interactions in CF lung disease. Regional differences of the microbiome in vivo were assessed in nine patients undergoing bronchoalveolar lavage (BAL) from three regions of the right lung jointly with protected brush sampling. The predominant taxa observed were similar between regions despite higher inflammation in the more diseased regions, as determined by concomitant CT, possible due to host-factors responsible for lung damage (Hogan et al., 307).

A rigorous longitudinal study addressed changes in microbiome related to exacerbations using culture-enriched metagenomics sequencing. Six patients contributed three sputum samples per week for a year. Diversity changed after exacerbations in some but not all patients without a consistent pattern or specific bacterial taxa contributing to changes (Whelan et al., 302 and 303). In contrast, a multi-center German study in 10 children using oral swab and sputum found that the presence of oral flora was associated with clinical stability. No consistent microbiome alterations occurred during exacerbations (Boutin et al., 323).

Metabolic activity of the microbiome remains challenging. Distinction of $\mathrm{CF}$ from non-CF sputum samples was more evident by host-derived sphingolipids than by Pseudomonas aeruginosa metabolites (Quinn et al., 308). Sputum from patients with stable lung function were found to have a more conservative metabolome, especially fewer antibiotic-resistance genes than those with declining lung function (Bacci et al., 306).

Nontuberculous mycobacteria (NTM) are ubiquitous in soil and water, yet airborne transmission can occur (Malcolm et al., 296). Detection of NTM remains challenging by culture and molecular methods. Various culture media for rapidly growing mycobacteria were compared (Preece et al., 376). The investigators concluded that their novel medium, "details to be published," had a 96-98\% detection rate compared to currently used methods ranging from $83 \%$ and $98 \%$ for different cepacia agars. In addition, the mycolic lipid acid barrier hinders lysis of mycobacteria for molecular detection. Even with an enhanced bacterial lysis protocol, the detection rate of NTM in spiked samples by sequencing was only $31 \%$ (Caverly et al., 372). 


\section{CLINICAL TRIALS}

The NACFC 2015 program highlighted ground-breaking results from clinical trials aimed at treating CFTR protein dysfunction. Data from post-approval studies of ivacaftor (VX-770) for people with the G551D and other gating mutations and the R117H mutation continue to add to our understanding of CFTR pathophysiology and restoration. Advances in microbiology, anti-inflammatories, and airway surface liquid restoration were also notable.

\section{CFTR Modulators}

The combination therapy lumacaftor (VX-809)/ivacaftor (Orkambi ${ }^{\circledR}$ ) was approved by the FDA in July 2015 for use in patients with CF ages 12 years of age and older with two copies of the F508del mutation. Two large phase III clinical trials ( $\mathrm{n}=1108$ subjects), TRAFFIC and TRANSPORT, demonstrated clinical benefit for patients on lumacaftor/ivacaftor with improvement in lung function (mean $\mathrm{FEV}_{1} \%$ predicted improvement 2.6-4.0\%) and reduction in pulmonary exacerbations of 30-39\% compared to the placebo group. ${ }^{4}$ Patients completing TRAFFIC and TRANSPORT were eligible for roll-over into PROGRESS, a 96week, open-label study of lumacaftor/ivacaftor; interim data from week 24 (48 weeks from initial study enrollment) were presented (Konstan et al., 211). Reassuringly, patients enrolled in PROGRESS maintained improvements in lung function seen in TRAFFIC and TRANSPORT. Improvements in body-mass index (BMI), Cystic Fibrosis QuestionnaireRevised (CFQ-R) respiratory domain health-related quality-of-life score, and rates of pulmonary exacerbation similar to those seen in the original trials were also maintained. Patients initially in the placebo group had similar and sustained $\mathrm{FEV}_{1}$ improvements with lumacaftor/ivacaftor treatment. There were no new safety concerns identified.

A phase III open-label study of ivacaftor in children ages 2-5 years with a gating mutation, the KIWI study, resulted in approval of ivacaftor for this age group. ${ }^{5}$ A 1-year follow-up study, KLIMB, demonstrated sustained improvements in fecal elastase and immunoreactive trypsinogen similar to those seen in KIWI over 72 weeks (Rosenfeld et al., 247). Notably, $8 / 33$ patients had elevations in liver enzymes $>8$ times the upper limit of normal. Six of the eight patients were able to restart therapy after a drug interruption, with two permanently discontinuing drug due to liver function abnormalities, confirming the importance of careful monitoring of liver enzymes in young children treated with ivacaftor.

Real-world experience with ivacaftor for patients with G551D and other gating mutations was presented by several groups. The impact of ivacaftor on mortality and lung transplantation in the 2 years following FDA approval was examined using CF Foundation Patient Registry (CFFPR) data (Bai et al., 246). Using data from all patients on ivacaftor (n =999) plus age and gender-matched controls $(n=4932)$, the researchers found a decreased relative risk of death (RR $0.37,95 \%$ CI $0.15-0.93$ ) and lung transplantation (RR 0.19, 95\% CI 0.05-0.66) in those on ivacaftor compared to controls. Hospitalization rates for all causes and pulmonary exacerbations were also lower in those on ivacaftor. In a separate study of patients with severe lung disease $\left(\mathrm{FEV}_{1}<40 \%\right.$ predicted) followed for a median of 3 years, treatment with ivacaftor $(\mathrm{n}=21)$ was associated with a reduced rate of death or lung transplantation (HR 0.13, CI 0.04-0.43) compared to controls $(\mathrm{n}=35)$ (Barry et al., 226). 
These studies confirm the substantial clinical impact of ivacaftor suggested by earlier clinical trials.

Several smaller studies also examined clinical outcomes after ivacaftor treatment. A single center study of patients in Ireland with the G551D mutation (Ronan et al., 205 and 206) showed improvements in $\mathrm{FEV}_{1}(+10.3 \%)$, BMI $\left(+1.2 \mathrm{~kg} / \mathrm{m}^{2}\right)$, sweat chloride $(-58 \mathrm{mmol} / \mathrm{L})$, and decreased rate of pulmonary exacerbations after initiation of ivacaftor in 33 patients. Improvements in chest CT score and decreased systemic inflammation (IL-6 and C-reactive protein) were also found. In a sub-group of 18 adults, treatment with ivacaftor did not result in changes in fecal elastase or gut inflammation; however, gut microbiota shifted with an increase in taxa typically associated with improved health. In a study from Toronto, changes in spirometry and lung clearance index (LCI) measured using multiple breath washout were compared in 10 patients with gating mutations before and after ivacaftor (Kane et al., 232). Over a median 37 days, LCI improved significantly with a decrease of $-2.3(-3.1$ to -1.4$)$, while $\mathrm{FEV}_{1}$ increased $12 \%$. There was no significant correlation between $\mathrm{FEV}_{1}$ and LCI changes.

KONDUCT, a randomized, placebo-controlled clinical trial which led to ivacaftor approval for people with $\mathrm{R} 117 \mathrm{H}$, included 69 patients ages $\ 6$ years treated for 24 weeks. ${ }^{6}$ Patients treated with ivacaftor had decreased sweat chloride and improved CFQ-R respiratory domain scores; lung function was improved in adults, but not in children. The G551D Observational Expanded and Extended Study (GOAL-E2) is a post-approval longitudinal study of patients before and after initiation of ivacaftor. Data from a subgroup analysis of CF patients ages $\checkmark 6$ years with R117H who started $(n=60)$ or did not start $(n=20)$ ivacaftor were presented (Sagel et al., 190). Patients who started ivacaftor were generally older, although 22/60 patients were under 18 years. Improvements in $\mathrm{FEV}_{1} \%$ predicted and CFQ$\mathrm{R}$ and decreases in sweat chloride over 6 months were similar to those seen in the clinical trial, regardless of age or poly-T tract status.

Data from early clinical trials of N91115, an oral S-nitrosoglutathione reductase (GSNOR) inhibitor with potential CFTR-modulatory and anti-inflammatory effects, were presented. A phase 1 pharmacokinetic open-label study of N91115 given over 14 days to CF subjects showed that the drug was safe and well-tolerated with overall exposure similar to previous findings in healthy subjects (Taylor-Cousar et al., 250). A phase Ib double-blind, randomized, placebo-controlled trial comparing three doses of N91115 in adults with CF homozygous for F508del $(\mathrm{n}=48)$ found no safety concerns over 1 month and a modest decrease in sweat chloride in the highest dose group (Donaldson, 270). A phase II study in adults with CF began enrollment in November 2015 (clinicaltrial.gov NCT02589236). This trial is unique in that $\mathrm{N} 91115$ is being studied in combination with lumacaftor/ivacaftor with the expectation that improved CFTR trafficking and stability due to increased Snitrosoglutathione will further augment the impact of lumacaftor/ivacaftor on CFTR function. ${ }^{7}$

\section{Gene Therapy}

Results from a Phase IIb double-blind, placebo-controlled trial of nebulized CFTR geneliposome complex were presented and subsequently published (Alton et al., 192). ${ }^{8}$ The 
study, part of the UK CF Gene Therapy Consortium translational program, enrolled $140 \mathrm{CF}$ patients ages 12 years and older from 18 sites and randomized patients to monthly nebulization with the gene-liposome complex or $0.9 \%$ saline for 12 months (clinicaltrials.gov NCT01621867). There was a statistically significant difference in the primary outcome, $\mathrm{FEV}_{1} \%$ predicted at 12 months of $3.7 \%$ (95\% CI $0.1-7.3, P=0.046$ ), largely due to a more rapid decline in $\mathrm{FEV}_{1}$ in the placebo group ( $-4.0 \%, \mathrm{CI}-6.6$ to -1.4$)$ compared to relative stability in the treatment group $(-0.4 \%, \mathrm{CI}-2.8$ to 2.1$)$. The study found no major safety concerns. Overall the benefits were modest, and the investigators suggested that further improvements with more potent and efficient gene vectors for delivery are needed before gene therapy may be suitable for clinical care.

\section{Microbiology}

Results from three clinical trials focused on treatment of methicillin-resistant

Staphylococcus aureus (MRSA) were presented. The STAR-too trial, enrolling patients with new onset MRSA infection, compared use of a rigorous combination therapy to observation alone, that is, standard of care. The primary endpoint of MRSA-culture negativity at day 28 was met by $82 \%$ in the treatment group compared to $26 \%$ in the observation arm (Goss, S11.3). Typing of the MRSA isolates from the trial showed a shift from the SCCmec II in prior studies to predominance of SCCmec IV type (USA300). Nasal colonization occurred in 32\% of participants but skin colonization was rare (Muhlebach et al., 360). The recently completed 20-center double-blind, placebo-controlled, Phase II study of vancomycin hydrochloride inhalation powder (AeroVanc ${ }^{\mathrm{TM}}$ ) enrolled 87 patients with chronic MRSA and met the primary endpoint of reduced MRSA colony forming units in sputum at day 29 (Dasenbrook, S11.4). Bronchoconstriction was observed at a higher rate in the AeroVanc ${ }^{\mathrm{TM}}$ group and appeared dose-dependent. The two-center Persistent MRSA-Eradication Protocol (PEMP)-blinded, placebo-controlled trial used the intravenous formulation of vancomycin for inhalation (Dezube et al., 327) in combination with oral TMP-SMX and rifampin. Among the 16 subjects who initiated study drug, three patients were withdrawn for bronchospasm. Rifampin resistance developed in five subjects. A report from France (Rubira et al., 330) described 15 patients with glycopeptide-intermediate-resistant MRSA (GISA) with a high rate of persistence. Treatment protocols used either prior, or after appearance of GISAwere too variable to allow evaluation of GISA risk factors and outcomes. Jointly such reports indicate that antibiotics should be used cautiously for MRSA.

Liposomal amikacin for inhalation (LAI) is a novel formulation of amikacin developed for people with $\mathrm{CF}$ and chronic $P$. aeruginosa or nontuberculous mycobacterial infections. ${ }^{9}$ An initial clinical trial, CLEAR-108, enrolled patients with CF age $\geq 6$ years with chronic $P$. aeruginosa infection. Participants were randomized to receive 3-monthly cycles of either LAI once daily or tobramycin inhalation solution (TIS) twice daily. Initial data showed noninferiority of LAI compared to TIS (Bilton et al., NACFC 2013, Abstract $235^{10}$ ). Eligible patients were able to roll-over to an open-label study of LAI, CLEAR-110, for 12 additional cycles over 2 years (Konstan et al., 212). Of the 206 patients enrolled, 134 completed 12 cycles of LAI; 44 prematurely discontinued the drug. After 12 cycles of LAI, participants had minimal change in $P$. aeruginosa density compared to baseline, pretreatment levels. 
Absolute $\mathrm{FEV}_{1}$ increased $\sim 3.5 \%$ but $\mathrm{FEV}_{1} \%$ predicted declined $\sim 3 \%$. Side effects were primarily respiratory and unrelated to study drug.

\section{Anti-Inflammatory Treatment}

CTX-4430 is a small molecule inhibitor of leukotriene A4 hydrolase that acts by reducing the inflammatory mediator leukotriene B4. A phase I safety study of ascending doses was performed in $17 \mathrm{CF}$ patients over 2 weeks (Ahuja et al., 287). The drug appeared safe and well-tolerated with no deleterious effects on lung function, and no changes in circulating neutrophils or airway bacterial load. There were trends noted in reduction of sputum neutrophils, elastase, and blood CRP, including a $65 \%$ reduction in sputum neutrophils in the highest dose group. A phase II trial is currently recruiting adults 18 years and older with lung function $250 \%$ (clinicaltrials.gov NCT 02443688).

\section{Restoration of the Airway Surface Liquid Layer}

$\mathrm{P}-1037$ is an $\mathrm{ENaC}$ inhibitor thought to aid in mucus hydration and mucus clearance in CF. In a Phase I safety study, healthy subjects $(\mathrm{n}=73)$ were enrolled in a randomized, doubleblind, placebo-controlled study of dose escalation of P-1037 and placebo (nebulized saline); a CF cohort ( $\mathrm{n}=8,2$ on placebo) was also evaluated (Donn et al., 217). Potassium levels and urine concentrations were monitored due to the potential impact of ENaC inhibitors on renal electrolyte transport and resultant hyperkalemia. ${ }^{11}$ At the highest dose studied, there were no changes in plasma $\mathrm{K}$ levels and drug excreted in the urine was very low; no adverse respiratory effects were noted. A phase II trial is now planned for P-1037 (renamed VX371) in combination with hypertonic saline.

\section{CLINICAL MANAGEMENT ISSUES}

\section{Imaging}

Advances in established chest $\mathrm{CT}$ methods for detecting structural lung disease using the PRAGMA scoring system as a more sensitive approach were reviewed in the "Assessing Presymptomatic Children” symposium (Rosenow, S10.2). ${ }^{12}$ PRAGMA-CF chest CT scores for gas trapping were not associated with air trapping on lung function or multiple breath washout, suggesting non-ventilatory defects (Rosenow et al., 216). While methodological improvements have reduced the radiation dose from chest CT, non-radiation-based imaging methods, such as MRI, have also seen increasing use in CF. In 57 children with CF, MRI scores differentiated those with chronic $P$. aeruginosa infection from those without; MRI scores also decreased after intravenous antibiotic treatment of a CF pulmonary exacerbation (Stahl et al., 209). Newer MRI-based technologies are also finding utility in CF. Ultra-short echo time (UTE) MRI revealed lung structural abnormalities that correlated with those observed on CT, as well as with ventilation defects seen on hyperpolarized gas MRI in five adolescents with CF (Thomen et al., 403). Similar findings were reported by a second group (Roach et al., 417). While promising, these newer MRI technologies will require substantial further development before they are ready to be used as research or clinical outcome measures. 


\section{Infection}

Numerous investigators demonstrated progress in management of $P$. aeruginosa, $S$. aureus, and other pathogens.

Current recommendations by European and the US guidelines are use of inhaled tobramycin as first line of therapy for $P$. aeruginosa. ${ }^{13,14}$ The approach to patients who fail to eradicate is still unresolved (Ratjen, S3.2). Persistence of $P$. aeruginosa could be secondary to host or bacterial factors. Using $P$. aeruginosa isolates obtained during the Early Pseudomonas Infection Control (EPIC) trial, isolates that persisted show higher rates of genetic mutations during the first year of infection compared to chronic infection (Jorth et al., 300).

Eradication strategies are associated with decreasing incidence of chronic $P$. aeruginosa as demonstrated using data from the CFFPR (Crull et al., 385). In patients $\geq 13$ years, the incidence of chronic $P$. aeruginosa, defined as $250 \%$ of cultures being positive, decreased from $14.3 \%$ in 2003 to $6.4 \%$ in 2012. In this epidemiologic study, treatment details were not reported. A prospective study of children born after 2002 and followed for a median of 5.1 years $(\mathrm{n}=6,277)$ addressed the current incidence of $P$. aeruginosa and the transition to chronic and mucoid infection (Khan et al., 294). Fifty-eight percent acquired $P$. aeruginosa at a median age of 1.1 years, and $94 \%$ of initial isolates were non-mucoid. Of the $27 \%$ of subjects progressing to chronic infection, mucoid $P$. aeruginosa was present in $18 \%$ at a mean (SD) 1.8 (2.2) years after initial infection. Initial mucoid infection was associated with higher risk of persistent infection.

Novel epidemiological and in vitro data address the choice of antibiotics for $P$. aeruginosa treatment. A multi-center study extended prior observations of potential interference of azithromycin with inhaled tobramycin (Happoldt et al., 292). ${ }^{15}$ Using data from a clinical trial comparing efficacy of TIS to inhaled aztreonam, patients on combination TISazithromycin had no improvement in lung function and a decrease in quality-of-life questionnaires compared to those on inhaled aztreonam or those on single therapy TIS. In vitro studies using clinical $P$. aeruginosa isolates grown in a biofilm model demonstrated reduced bacterial killing and induction of aminoglycoside efflux pumps by azithromycin as potential mechanisms. A prospective clinical trial examining the effect of combining azithromycin with inhaled tobramycin for patients with $\mathrm{CF}$ and chronic $P$. aeruginosa is planned (clinicaltrials.gov NCT02677701). The effect of inhaled antibiotics on bacterial killing was also studied using clinical isolates from patients on (i) alternating months TIS; (ii) alternating months inhaled aztreonam; or (iii) alternating TIS and aztreonam (Shih et al., 366). In general, aztreonam failed to reduce biofilms, whereas TIS appeared more effective. Subjects on alternating antibiotics had the lowest resistance.

\section{Psychosocial Issues}

Elevated rates of anxiety and depression among adolescents and adults with $\mathrm{CF}$ and their parental caregivers may affect adherence, health outcomes, health care utilization and cost. 16-18 The importance of mental health was highlighted in a plenary session entitled, "There is No Health Without Mental Health," (Quittner, Elborn and Smith, P3) which offered a comprehensive review of mental health issues in $\mathrm{CF}$ and introduced guidelines established 
by the Cystic Fibrosis Foundation and the European Cystic Fibrosis Society. ${ }^{19}$ These guidelines call for annual screening of adolescents and adults for depression and anxiety, with appropriate interventions and reassessment.

Adherence to CF therapies impacts outcomes, and the relationship between disease severity, treatment complexity, and psychosocial concerns are important areas of study in CF. Factors linking socioeconomic status and respiratory outcomes in children and the need for appropriate resources to support adherence were explored (Oates et al., 661). ${ }^{20}$ Strategies to improve adherence to CF therapies include promotion of healthy lifestyles (Wilcox et al., 623), use of home spirometry and symptom diaries (Lechtzin et al., 628), inpatient medication self-administration (Khan et al., 624), and attention to spiritual needs (Grossoehme et al., 637).

Adolescents and young adults must assume increasing responsibility for their own health care as they prepare to transition from pediatric to adult CF care. Extensive efforts are underway to improve the transition process, including structured and mentored quality improvement (QI) programs (Baker et al., 640). ${ }^{21}$ Quality improvement initiatives from individual centers involve use of transition checklists, combined pediatric and adult team meetings, provider handoffs, and transition-specific educational materials (Burns et al., 610; McLauchlan et al., 611; Ruddy et al., 612; Becker et al., 613; Cumming et al., 614; Baum et al., 615).

Transition-related process of care was examined using the CFFPR (Sawicki et al., 486). Transfers of care from pediatric to adult providers most commonly occurred at age 20 with median of 99 days between last pediatric and first adult outpatient encounter. Patients with markers of better overall health during the last year of pediatric care had longer gaps in care. Minimal disruption in care occurred during transfer of individual patients from pediatric to adult CF care. In a separate study, a majority of adolescents and young adults indicated they would use social media for CF education, peer support, and communicating with health care providers (Anand et al., 663). Infection control restrictions support the need to enhance social media platforms.

Palliative care focuses on enhancing quality of life for people with serious illness. Funded research and center-based QI initiatives are yielding new information about advance care planning (ACP), end-of-life care, provider education, and other aspects of palliative care in $\mathrm{CF}$. Chen et al. ${ }^{22}$ reported that most adults with $\mathrm{CF}$, regardless of disease severity, are open to conversations with health care providers about goals of care and end-of-life care issues. Adolescents with advanced disease and their pediatric $\mathrm{CF}$ providers were also found to be receptive to ACP (Kazmerski et al., 666). Using data from the US adult CF care programs, Dellon et al. ${ }^{23}$ reported more ACP occurs now than in previous reports, but often late in the disease. There appears to be a wide variation in ACP practices among care centers.

Providing quality palliative care to patients with CF necessitates appropriate support and education for health care providers around palliative and end-of-life care issues. Goggin and Cohen found that even experienced CF care providers reported lacking preparation and knowledge in end of life care and desired more education. ${ }^{24}$ Linnemann et al. ${ }^{25}$ created and 
implemented a palliative care curriculum for $\mathrm{CF}$ care providers addressing supportive care resources, pain and symptom management, communication and psychosocial skills that improved self-assessed comfort level with core palliative care skills. Other centers reported observations from integrating specialty palliative care into the care of people with CF and suggested that, even with availability of expert consultation, palliative, and end-of-life care needs and practices specific to CF warrant further investigation (Liberman et al., 646;

Rabinowitz et al., 647).

\section{Quality Improvement}

Quality improvement is defined as an implementation of system changes that drive care processes and result in measurable improvement. In this section, we highlight some of the important clinical QI work presented at the 2015 NACFC meeting with a focus on interventions aimed to achieve systems of best practice, patient and family engagement, and use of registry data to identify areas for improvement. Lastly, we discuss future strategies for successful QI implementation.

Centers reported interventions that increased in-clinic services to improve patient convenience and guideline adherence (Ghazala et al., 524; Joshi et al., 534). At Nemours Children's Specialty Care, bundled intervention with in-clinic access to fasting glucose, preclinic identification of eligible patients, and CF nurse coordinator reminders led to an increase in screening for CF related diabetes from $36 \%$ to $86 \%$ of eligible patients (Smith et al., 543). Other centers used phone calls, reminder letters, and education of staff to better adhere to guidelines (McDonagh et al., 516; Collins et al., 523). A recent quality report described improved patient adherence to quarterly visits, cultures, and biannual lung function through a nursing-led appointment tracking system. ${ }^{26}$ Reporting important contextual information, such as the size of center, patient demographics, and team composition allows other centers to ascertain whether certain elements of these interventions may be applicable to their clinics.

Patient engagement and education was another key theme in reported interventions (Clark et al., 530; Finklea et al., 531). A multifaceted nutrition intervention program used patient questionnaires to assess perceived barriers to weight gain. With this knowledge, the group targeted high-risk patients for an incentive program and nutritional supplements, supported through local funding sources. Sixteen of 21 below BMI goal patients achieved $>90 \%$ of goal BMI and 29\% increased to BMI above goal (Robaczynski et al., 546).

Improving the partnership of providers and patients in the context of health care service was introduced in a symposium on "co-production" (Batalden, George, Phillips, Margolis, and Marshall, S12). The construct of co-production involves reflective communication with patients and allows for engagement that informs and designs systems of health care service and quality care. ${ }^{27}$ An example of this comes from the Cystic Fibrosis Trust (CFT) of the United Kingdom (UK) who surveyed the CF community to gauge interest and information for future designs of the UK CF registry (Cosgriff et al., 515). Using multiple social media methods to reach the community, the CFT acquired 848 individual patient and caregiver responses with $81 \%$ wishing to view their personal data and $94 \%$ willing to self-report information for patient experience and patient reported outcomes. 
CF-specific experience of care surveys have been developed and tested in the United States and Germany. ${ }^{28,29}$ An expansion of the CF Patient and Family Experience of Care Survey in 46 pediatric programs defined 1057 patient-provider relationships as personal (27\%), paternalistic (12\%), or as a partnership (10\%) (Homa et al., 542). Increasing partnership or personal relationships compared to paternalistic relationships was the predominant feedback to improve patient-provider relationships. Engaging active patient voice has the potential to shape our systems of care and provides the opportunity to individualize the experience of chronic disease.

Measuring the impact of QI interventions requires data to audit processes and outcomes of current practice. The CFFPR is an active resource to measure QI. Validity of CFFPR data was augmented with recently reported results of an internal data audit (Dowd et al., 538). At 48 care centers, data accuracy and completeness was $96 \%$ for patient-specific details including height, weight, lung function, and microbiology data but $75-80 \%$ for chronic medication use.

The CFFPR also provides a base for observational comparisons. Colleagues at St. Louis Children's Hospital used their center data to compare the nutritional status of a cohort of infants born between 2007 and 2010 with a newborn-screened cohort born between 2011 and 2014 who received an early nutritional intervention with intensive counseling, education, and follow-up (Clark et al., 530). Infants who received the intervention had significant improvements in weight-for-height z-score by 24 months of age ( -0.6 to $1.0, P$ $<0.001)$.

As new guidelines emerge and the pipeline of therapeutics matures, systems of care must be developed in parallel to facilitate the opportunity for all patients to achieve best clinical outcomes. System measurements, such as through the CFFPR or an electronic medical record, allow for dynamic feedback to improve care. Active family partnership and/or patient-level factors assessed through site-specific and experience surveys will enrich and direct personalized care. Similarly, comparative effectiveness trials can be used to evaluate both efficacy and patient experience. ${ }^{30}$ Publication of QI work should adhere to the standards of reporting guidelines to learn from the successes and challenges among care centers. ${ }^{31,32}$ Lastly, committed care teams must take up the challenge of developing and testing high quality care systems despite competing responsibilities. Support of clinical personnel should be a priority to aid in the acceleration of CF quality care (Walker et al., 529).

\section{CONCLUSIONS}

The 2015 North American Cystic Fibrosis Conference presented updates on many exciting new concepts and advances. Progress continues to be made in understanding the basic pathophysiology of CFTR, mucus, and infections in the CF lung, with implications for new treatment strategies. There has been impressive progress in clinical trials of new therapies that are likely to transform CF care in the near future. Clinical management of CF is evolving rapidly through application of QI methods, and increased attention to individualized patient goals and psychosocial factors. 


\section{Acknowledgments}

Funding source: Cystic Fibrosis Foundation, Numbers: ZEMANI12A0, NIH/NHLBI K23HL114883, CC030-14, DELLON14Q10, MUHLEB15A0, STARtoo10K0, NIH/NHLBI RFA-HL-12-035, NIH/NHLBI R01-HL116228, NIH/NIDDK P30-DK065988.

\section{References}

1. Pediatr Pulmonol; The 29th Annual North American Cystic Fibrosis Conference; 2015. p. 1-474.

2. Veit G, Avramescu RG, Chiang AN, Houck SA, Cai Z, Peters KW, Hong JS, Pollard HB, Guggino WB, Balch WE, et al. From CFTR biology toward combinatorial pharmacotherapy: expanded classification of cystic fibrosis mutations. Mol Biol Cell. 2016; 27:424-433. [PubMed: 26823392]

3. Xue X, Mutyam V, Tang L, Biswas S, Du M, Jackson LA, Dai Y, Belakhov V, Shalev M, Chen F, et al. Synthetic aminoglycosides efficiently suppress cystic fibrosis transmembrane conductance regulator nonsense mutations and are enhanced by ivacaftor. Am J Respir Cell Mol Biol. 2014; 50:805-816. [PubMed: 24251786]

4. Wainwright CE, Elborn JS, Ramsey BW, Marigowda G, Huang X, Cipolli M, Colombo C, Davies JC, De Boeck K, Flume PA, et al. Lumacaftor-ivacaftor in patients with cystic fibrosis homozygous for Phe508del CFTR. N Engl J Med. 2015; 373:220-231. [PubMed: 25981758]

5. Davies JC, Cunningham S, Harris WT, Lapey A, Regelmann WE, Sawicki GS, Southern KW, Robertson S, Green Y, Cooke J, et al. Safety, pharmacokinetics, and pharmacodynamics of ivacaftor in patients aged 2-5 years with cystic fibrosis and a CFTR gating mutation (KIWI): an open-label, single-arm study. Lancet Respir Med. 2016; 4:107-115. [PubMed: 26803277]

6. Moss RB, Flume PA, Elborn JS, Cooke J, Rowe SM, McColley SA, Rubenstein RC, Higgins M. Efficacy and safety of ivacaftor in patients with cystic fibrosis who have an Arg117His-CFTR mutation: a double-blind, randomised controlled trial. Lancet Respir Med. 2015; 3:524-533. [PubMed: 26070913]

7. Zaman K, Sawczak V, Zaidi A, Butler M, Bennett D, Getsy P, Zeinomar M, Greenberg Z, Forbes M, Rehman S, et al. Augmentation of CFTR maturation by S-nitrosoglutathione reductase. Am J Physiol Lung Cell Mol Physiol. 2016; 310:L263-L270. [PubMed: 26637637]

8. Alton EW, Armstrong DK, Ashby D, Bayfield KJ, Bilton D, Bloomfield EV, Boyd AC, Brand J, Buchan R, Calcedo R, et al. Repeated nebulisation of non-viral CFTR gene therapy in patients with cystic fibrosis: a randomised, double-blind, placebo-controlled, phase $2 \mathrm{~b}$ trial. Lancet Respir Med. 2015; 3:684-691. [PubMed: 26149841]

9. Ehsan Z, Clancy JP. Management of Pseudomonas aeruginosa infection in cystic fibrosis patients using inhaled antibiotics with a focus on nebulized liposomal amikacin. Future Microbiol. 2015; 10:1901-1912. [PubMed: 26573178]

10. Pediatr Pulmonol; The 27th Annual North American Cystic Fibrosis Conference Pediatr Pulmonol; 2013. p. 207-453.

11. O’Riordan TG, Donn KH, Hodsman P, Ansede JH, Newcomb T, Lewis SA, Flitter WD, White VS, Johnson MR, Montgomery AB, et al. Acute hyperkalemia associated with inhalation of a potent ENaC antagonist: phase 1 trial of GS-9411. J Aerosol Med Pulm Drug Deliv. 2014; 27:200-208. [PubMed: 23905576]

12. Rosenow T, Oudraad MC, Murray CP, Turkovic L, Kuo W, de Bruijne M, Ranganathan SC, Tiddens HA, Stick SM. Australian Respiratory Early Surveillance Team for Cystic Fibrosis (AREST CF). A quantitative structural lung disease computed tomography outcome in young children with cystic fibrosis. Am J Respir Crit Care Med. 2015; 191:1158-1165. [PubMed: 25756857]

13. Doring G, Flume P, Heijerman H, Elborn JS. Treatment of lung infection in patients with cystic fibrosis: current and future strategies. J Cyst Fibros. 2012; 11:461-479. [PubMed: 23137712]

14. Mogayzel PJ Jr, Naureckas ET, Robinson KA, Brady C, Guill M, Lahiri T, Lubsch L, Matsui J, Oermann CM, Ratjen F, et al. Cystic Fibrosis Foundation pulmonary guideline. Pharmacologic approaches to prevention and eradication of initial Pseudomonas aeruginosa infection. Ann Am Thorac Soc. 2014; 11:1640-1650. [PubMed: 25549030] 
15. Nick JA, Moskowitz SM, Chmiel JF, Forssen AV, Kim SH, Saavedra MT, Saiman L, Taylor-Cousar JL, Nichols DP. Azithromycin may antagonize inhaled tobramycin when targeting Pseudomonas aeruginosa in cystic fibrosis. Ann Am Thorac Soc. 2014; 11:342-350. [PubMed: 24476418]

16. Quittner AL, Goldbeck L, Abbott J, Duff A, Lambrecht P, Sole A, Tibosch MM, Bergsten Brucefors A, Yuksel H, Catastini P, et al. Prevalence of depression and anxiety in patients with cystic fibrosis and parent caregivers: results of the International Depression Epidemiological Study across nine countries. Thorax. 2014; 69:1090-1097. [PubMed: 25246663]

17. Snell C, Fernandes S, Bujoreanu IS, Garcia G. Depression, illness severity, and healthcare utilization in cystic fibrosis. Pediatr Pulmonol. 2014; 49:1177-1181. [PubMed: 24619910]

18. Quittner AL, Saez-Flores E, Barton JD. The psychological burden of cystic fibrosis. Curr Opin Pulm Med. 2016; 22:187-191. [PubMed: 26814144]

19. Quittner AL, Abbott J, Georgiopoulos AM, Goldbeck L, Smith B, Hempstead SE, Marshall B, Sabadosa KA, Elborn S, et al. International Committee on Mental Health. International Committee on Mental Health in Cystic Fibrosis: Cystic Fibrosis Foundation and European Cystic Fibrosis Society consensus statements for screening and treating depression and anxiety. Thorax. 2016; 71:26-34. [PubMed: 26452630]

20. Oates GR, Stepanikova I, Gamble S, Gutierrez HH, Harris WT. Adherence to airway clearance therapy in pediatric cystic fibrosis: socioeconomic factors and respiratory outcomes. Pediatr Pulmonol. 2015; 50:1244-1252. [PubMed: 26436321]

21. Baker AM, Riekert KA, Sawacki GS, Eakin MN. CF RISE: implementing a clinic-based transition program. Pediatr Allergy Immunol Pulmonol. 2015; 28:250-254.

22. Chen E, Killeen KM, Peterson SJ, Saulitis AK, Balk RA. Evaluation of pain, dyspnea, and goals of care among adults with cystic fibrosis: a comprehensive palliative care survey. Am J Hosp Palliat Care. 2016 pii:1049909116629135. [Epub ahead of print].

23. Dellon EP, Chen E, Goggin J, Homa K, Marshall BC, Sabadosa KA, Cohen RI. Advance care planning in cystic fibrosis: current practices, challenges, and opportunities. J Cyst Fibros. 2016; 15:96-101. [PubMed: 26362396]

24. Goggin J, Cohen RI. CF healthcare workers feel unprepared in providing suitable end of life care and desire more education: results of a nationwide survey. J Cyst Fibros. 2016; 15:85-89. [PubMed: 26362397]

25. Linnemann RW, O’Malley PJ, Friedman D, Georgiopoulos AM, Buxton D, Altstein LL, Sicilian L, Lapey A, Sawicki GS, Moskowitz SM. Development and evaluation of a palliative care curriculum for cystic fibrosis healthcare providers. J Cyst Fibros. 2016; 15:90-95. [PubMed: 25817162]

26. Wooldridge JL, Mason S, Brusatti J, Albers GM, Noyes BE. Improvements in cystic fibrosis quarterly visits, lung function tests, and respiratory cultures. Pediatrics. 2015; 136:1611-1616.

27. Batalden M, Batalden P, Margolis P, Seid M, Armstrong G, Opipari-Arrigan L, Hartung H. Coproduction of healthcare service. BMJ Qual Saf. 2015; doi: 10.1136/bmjqs-2015-004315

28. Homa K, Sabadosa KA, Marrow LC, Marshall BC. Experience of care from the perspective of individuals with cystic fibrosis and families: results from $70 \mathrm{CF}$ Foundation accredited programs in the USA. J Cyst Fibros. 2015; 14:515-522. [PubMed: 25649286]

29. Steinkamp G, Stahl K, Ellemunter H, Heuer E, van Konings-bruggen-Rietschel S, Busche M, Bremer W, Schwarz C. Patient Experience Working Group. Cystic fibrosis (CF) care through the patients' eyes - a nationwide survey on experience and satisfaction with services using a diseasespecific questionnaire. Respir Med. 2015; 109:79-87. [PubMed: 25516453]

30. Sawicki GS, Goss CH. Tackling the increasing complexity of CF care. Pediatr Pulmonol. 2015; 50:S74-S79. [PubMed: 26335957]

31. Ogrinc G, Davies L, Goodman D, Batalden P, Davidoff F, Stevens D. SQUIRE 2.0 (Standards for QUality Improvement Reporting Excellence): revised publication guidelines from a detailed consensus process. BMJ Qual Saf. 2015; doi: 10.1136/bmjqs-2015-004411

32. Boyle MP, Sabadosa KA, Quinton HB, Marshall BC, Schechter MS. Key findings of the US Cystic Fibrosis Foundation's clinical practice benchmarking project. BMJ Qual Saf. 2014; 23:i15-i22. 